\title{
Research on the application of change detection technology based on multisource remote sensing images in the dynamic change of land use
}

\author{
Xiang Zhang ${ }^{1, a, *}$, Maofa Wang ${ }^{1, b}$ \\ ${ }^{1}$ Department of Information Technology, Institute of Disaster Prevention, Yanjiao, Sanhe, China \\ a. archy@aliyun.com, b. wangmaofa2008@126.com \\ *corresponding author
}

Keywords: Remote sensing image, False color images, Change detection technology, Land use change.

\begin{abstract}
When the earthquake occurs, the damage near the active fault zone will be particularly serious. So it is necessary to find out the exact direction and width of the main active fault zone, and the change of land surface use. Several key areas were selected from Xiadian active fault zone, and the remote sensing data for several consecutive years in these key areas were analyzed. By using the change detection model of remote sensing images on active fault zone, the changes of various land factors in these key areas were clarified, to provide an important basis for ensuring the safety of people's lives and property and carrying out urban land planning in a scientific way.
\end{abstract}

The development of satellite technology allows us to obtain detailed information about the large scale of land surface on the typical fault zone, get rid of the traditional method's shortcomings, such as long-time monitoring and the difficulty of locating the specific boundaries, and overcome the difficulties in the past that it is difficult to consider the land use situation as a whole from the time and space[1-3].The paper aimed on detecting the land use change of active fault zone key area. Taking Xiadian as an example, the change detection technology based on multisource remote sensing images was studied.

\section{$2 \quad$ Remote Sensing Images Processing}

The data of this paper mainly came from remote Sensing data Center of Maryland University, and its website is http:/glcf.umiacs.umd.edu/data/. Part of the data came from Earth Google. The data acquisition time spanned from 2006 to 2011, which mainly came from Landsat5, Landsat7 remote Sensing satellites. 
The remote sensing images used in this paper are multi-bands storage files, which are the combine of the seventh, fourth and third band files. Water body is deep-blue which is easier to be identified out in the fusion images[4-6].

\section{The Automatic Change Detection Methods}

The paper used automatic change detection method based on probability statistical model, which mainly includes 4 steps: texture image synthesis, difference image acquisition, extraction of change target or area, and detection precision analysis[7-9].

Texture acquisition method uses gray level co-occurrence matrix to describe the image texture. Gray level co-occurrence matrix (GLCM) is a statistical form of the joint distribution of two gray level pixels, and can well reflect the correlation rule of the texture gray level. The each element value of the gray level co-occurrence matrix is determined by the following formula:

$$
p_{i, j}=\frac{p(\mathrm{i}, \mathrm{j}, \mathrm{d}, \theta)}{\sum_{i} \sum_{j} p(\mathrm{i}, \mathrm{j}, \mathrm{d}, \theta)}
$$

In the formula, $p(\mathrm{i}, \mathrm{j}, \mathrm{d}, \theta)$ is occurrences of a pair of image points whose grey are respectively $\mathrm{i}$ and $\mathrm{j}$, distance is $\mathrm{D}$ and the direction is $\theta$.

The change detection method used in the difference image is based on the $t$ test in mathematical statistics, by analyzing the relationship between means and variance of the different remote sensing images in a special neighborhood from different time to determine its difference. The $t$ value of each pixel $t$ reflects the degree of change.

Change target extraction divides the image into two types: the change and non-change, so it is considered as a classification problem. It uses threshold method to distinguish the change class and the non-change class.

The evaluation method in detection precision analysis mainly uses error matrix, and related evaluation indicators include: overall classification accuracy, user accuracy, production accuracy, Kappa coefficient.

\section{Interpreting Major Land Use Change in Xiadian Fault Zone}

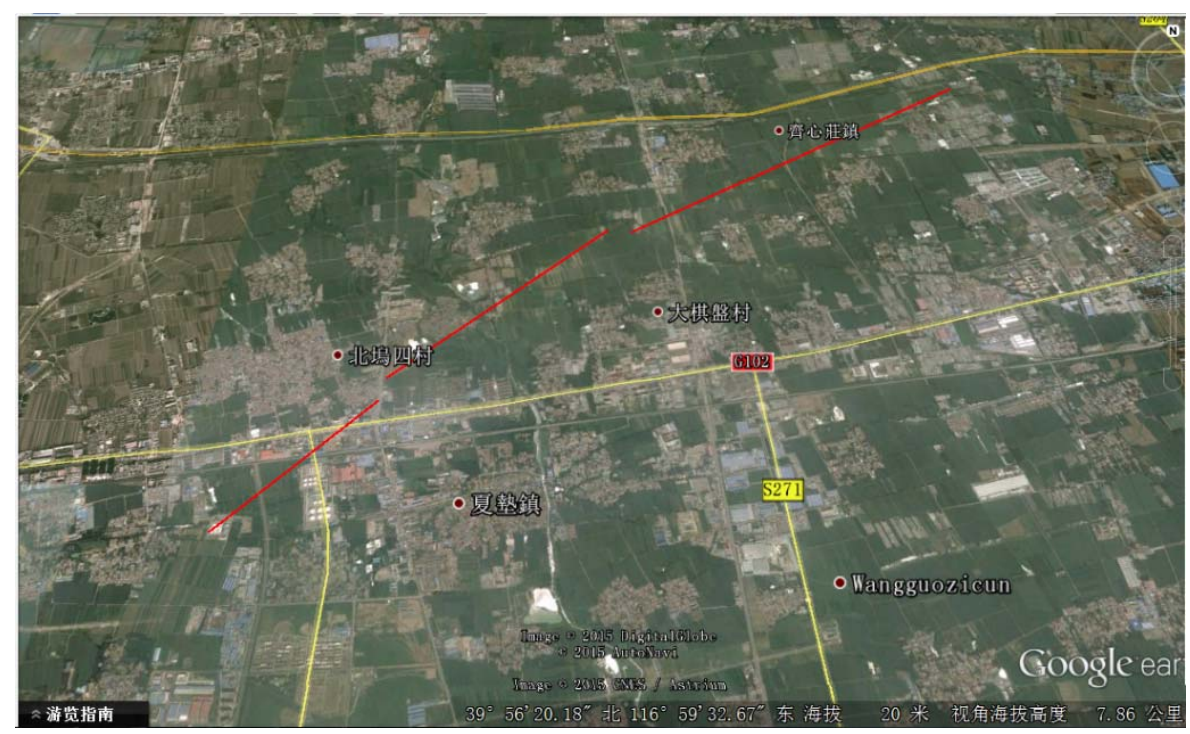

Figure 1: Xiadian fault zone 
Figure 1 is a remote image of Xiadian fault zone. The fault zone passes through 102 National Highway and is marked by a red line. The area is nearby the capital Beijing, and its economic conditions, transportation, and culture are developed. The change of land use is rapid and frequent. Near the fault zone marked by the red line, 19 geographic coordinate points were chosen as land use change detecting areas.

Due to space limitations, this paper only described the analysis process of one point in Xiadian fault zone, whose longitude and latitude coordinates are $116^{\circ} 54^{\prime} 27.88^{\prime \prime}, 39^{\circ} 57^{\prime} 18.10^{\prime \prime}$, and its altitude is 21 meters.

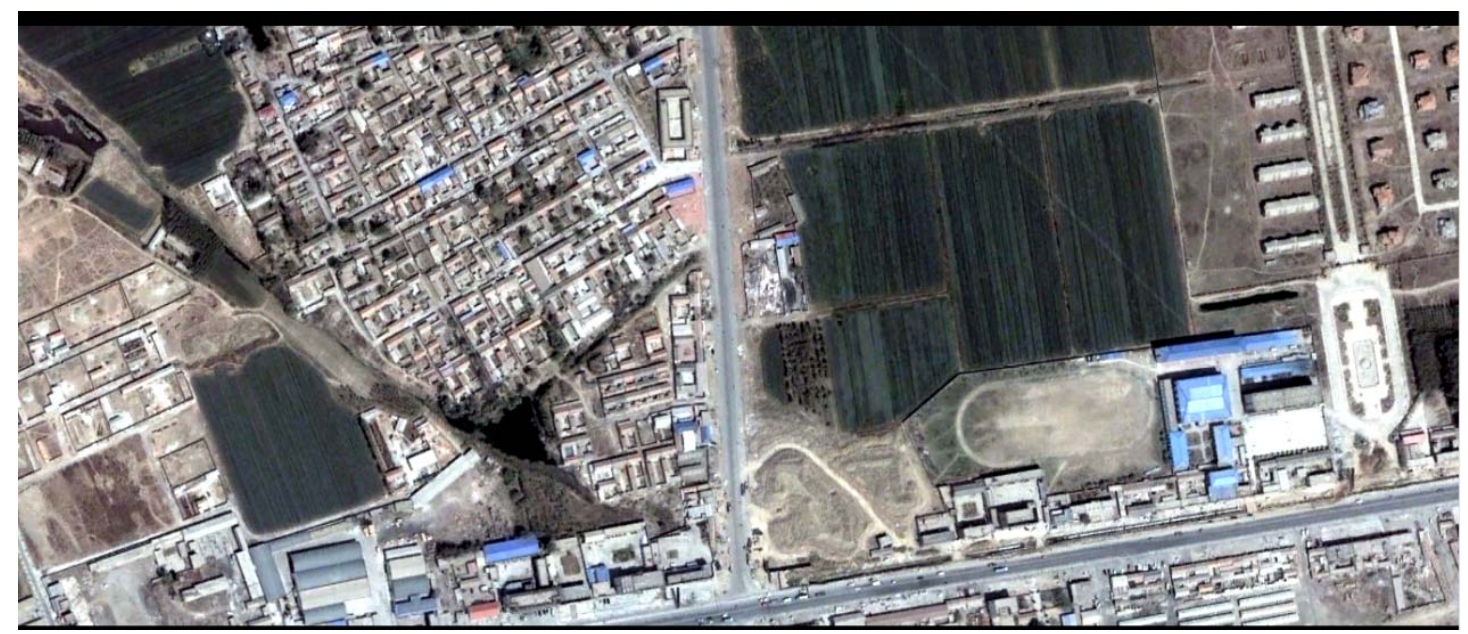

Figure 2: The false color image synthesized by the 7th, 4th, 3rd bands on May 2th, 2006

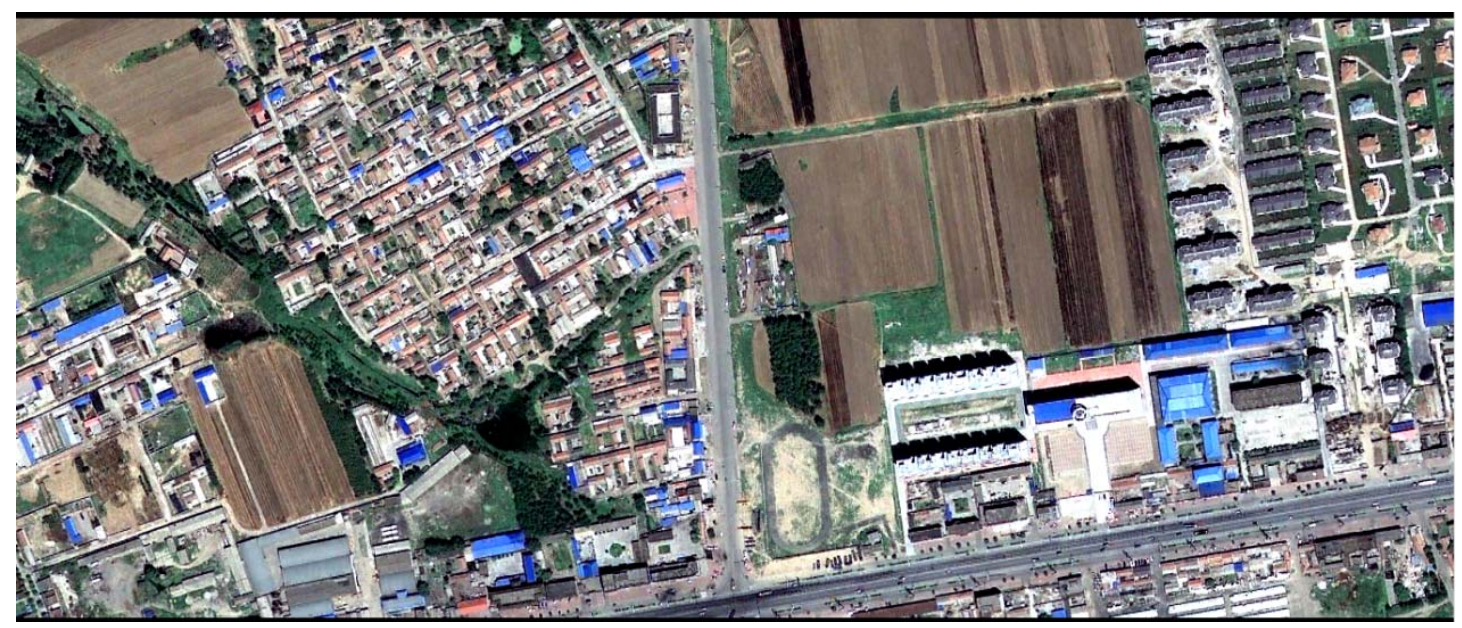

Figure 3: The false color image synthesized by the 7th, 4th, 3rd bands on June 28th, 2009 


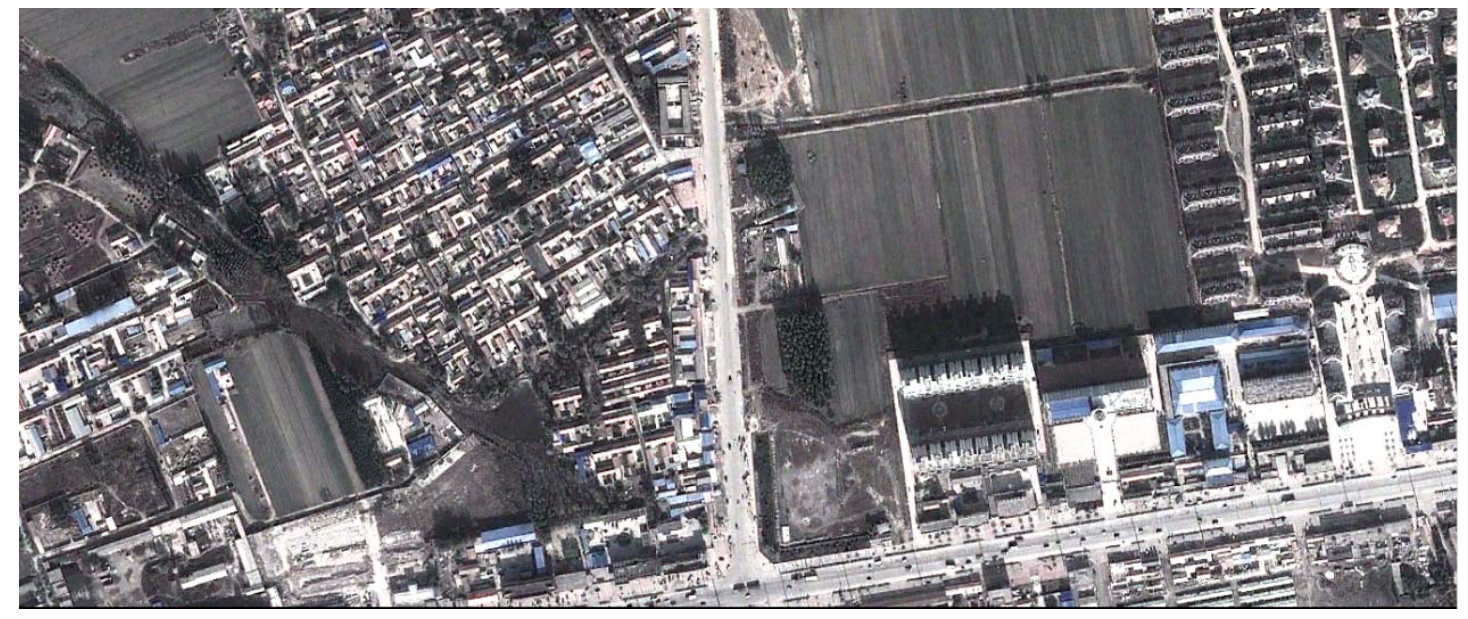

Figure 4: The false color image synthesized by the 7th, 4th, 3rd bands on October 27th, 2010

Figure 2, Figure 3 and Figure 4 are three images respectively corresponding to three years: 2006, 2009,2010 . As shown in the three figures, housing on the left side of the region gradually increased, green was also getting better and better, central farmlands had been abandoned, some of the waste land was used to build the factory, and new constructions appeared on the right side of the region.

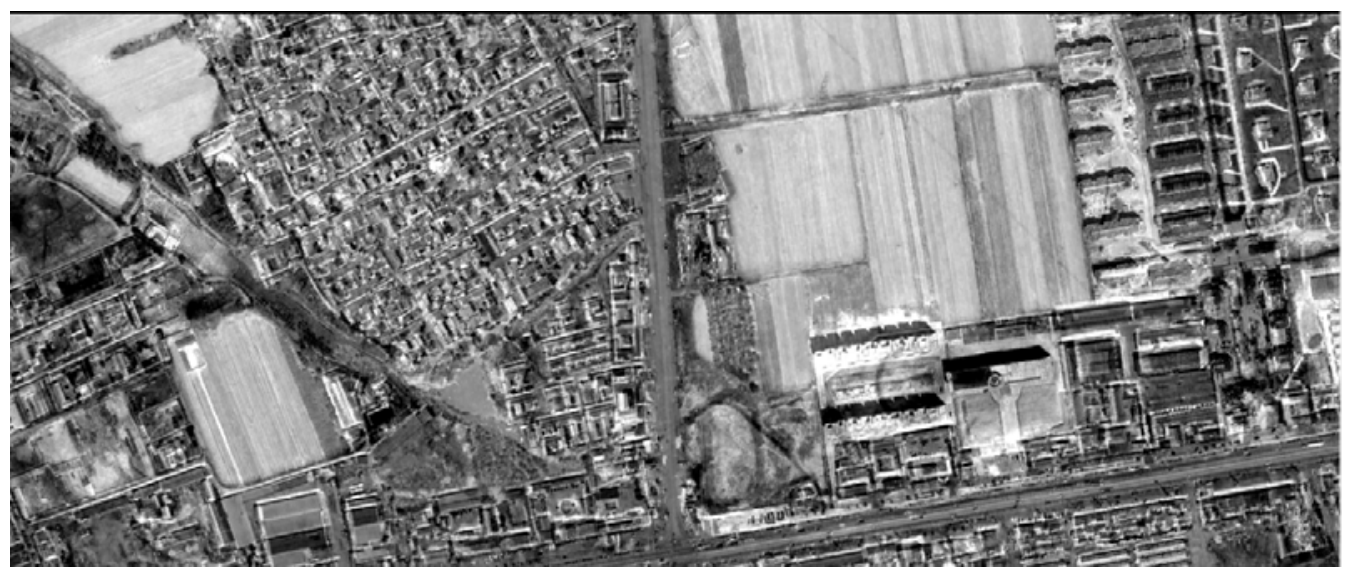

Figure 5: Figure 2 and Figure 3's different change file

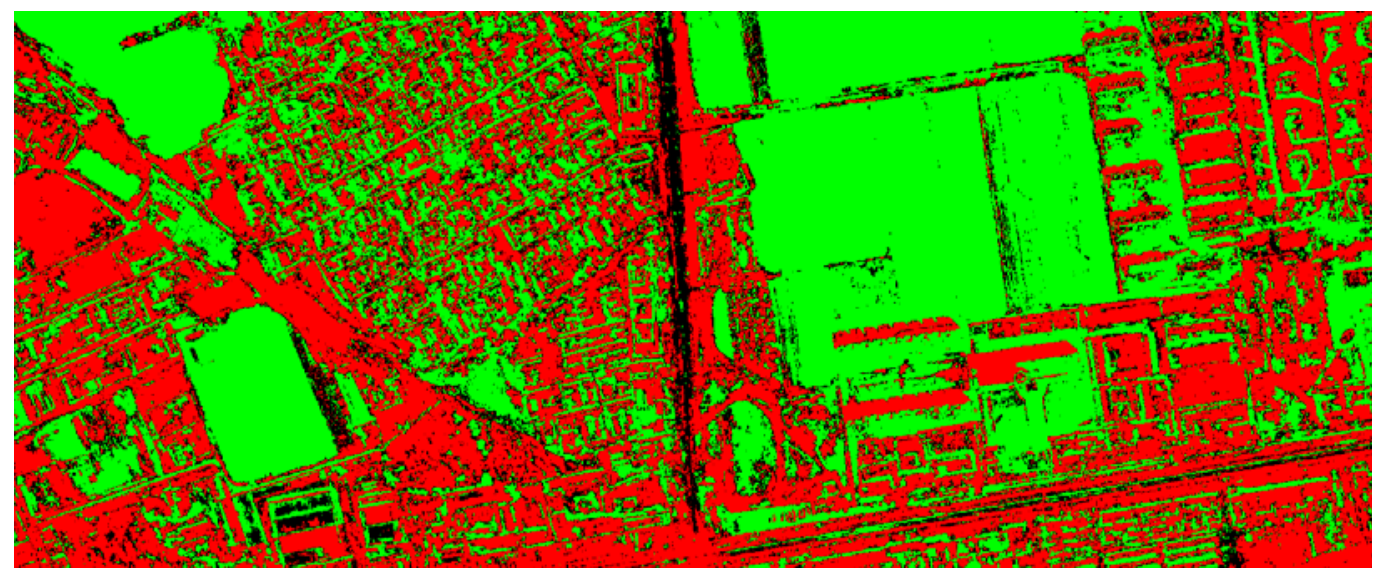

Figure 6: Figure 2 and Figure 3's highlight change file 
Figure 5, Figure 6 are different change and highlight change files between year 2006 and year 2009 , respectively.

In Figure 5 the gray value of farmland area had a larger change, while in Figure 6 it decreased, which indicated these farmland had not been used in time, may be abandoned. In Figure 5 the gray value of the farmland near the right region changed distinctly, while in Figure 6 it increased. The cause may be more green appeared and construction or expansion of urban housing. As shown in Figure 6, the gray value on both sides of the road represented in black area obviously increased, which illustrated the greening on both sides of the road was better.

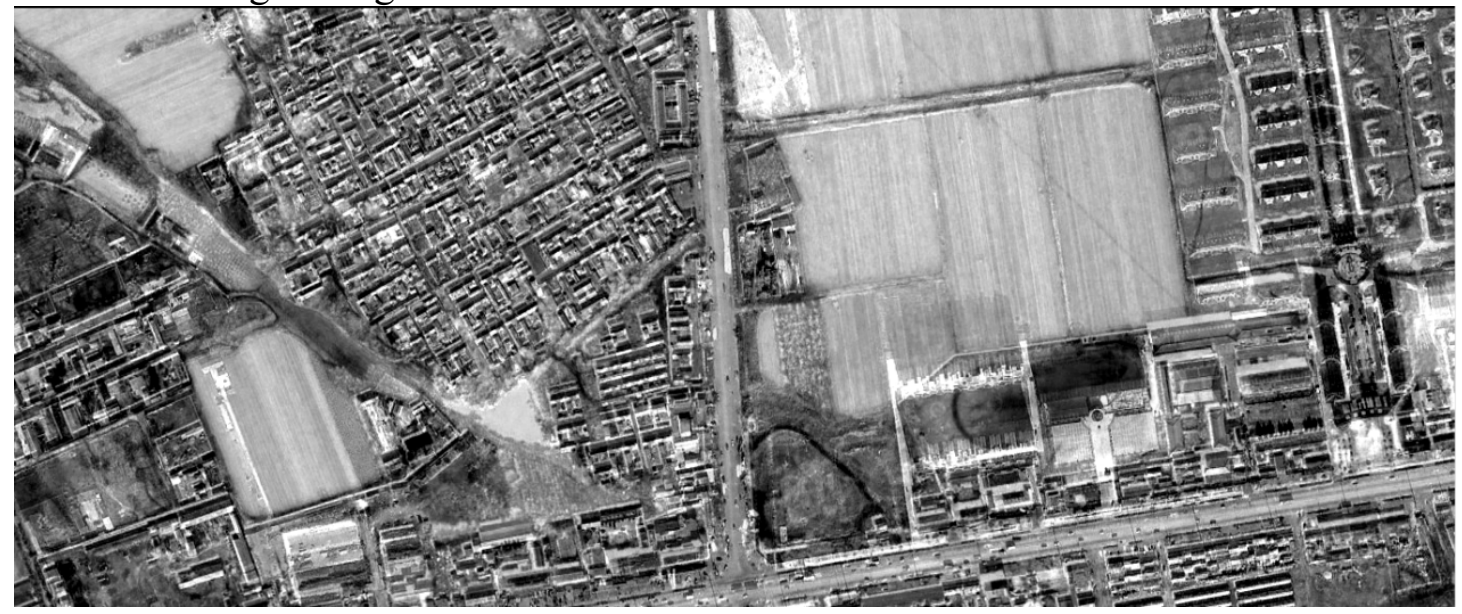

Figure 7: Figure 2 and Figure 4's different change file

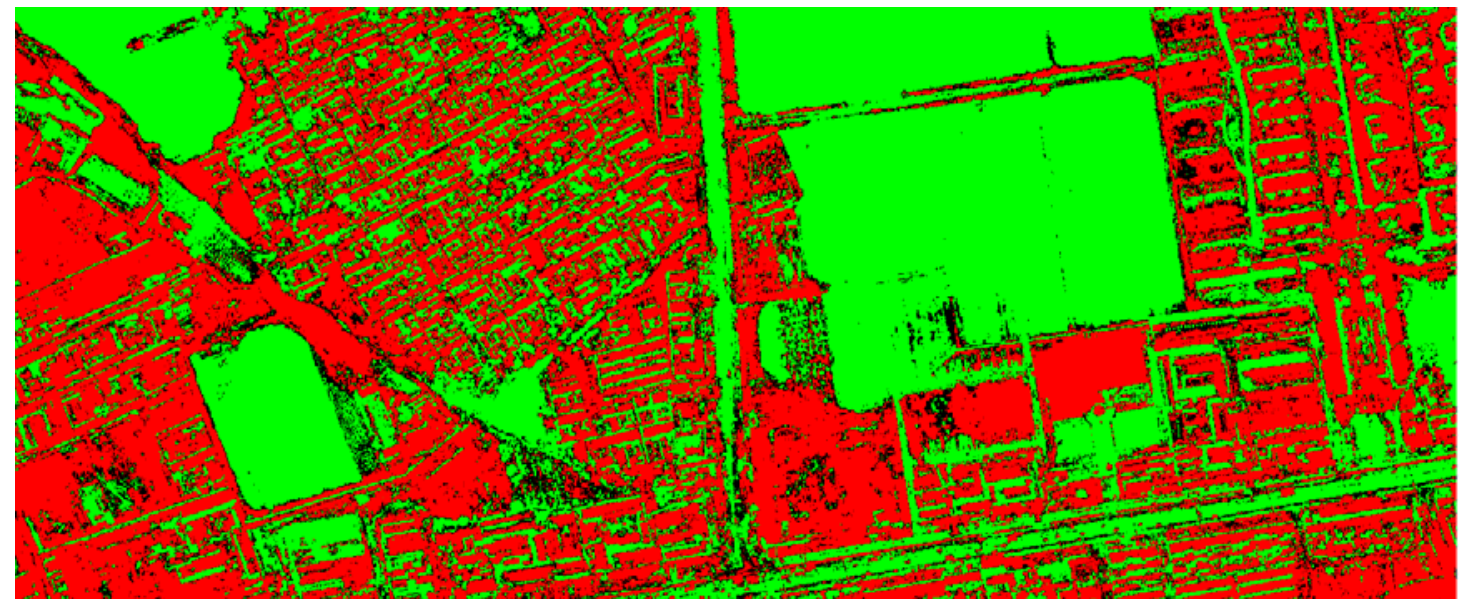

Figure 8: Figure 2 and Figure 4's highlight change file

Figure 7, Figure 8 are different change and highlight change files between year 2006 and year 2010 , respectively.

It can be seen from the comparison of Figure 7 and Figure 9 that the area of farmland was smaller; the gray value of farmland changed little while the gray value of farmland surrounding area changed much. As shown in Figure 8, the gray value of farmland decreased, and the gray value of farmland surrounding area increased. The reasonable explain was that the farmland was still not used in time and idle; the farmland surrounding area was partially occupied, and the new buildings were built. It illustrated the green level in residential areas and factories was getting higher and higher that the grey value of constructions changed greatly in Figure 7 and increased in Figure 8. 


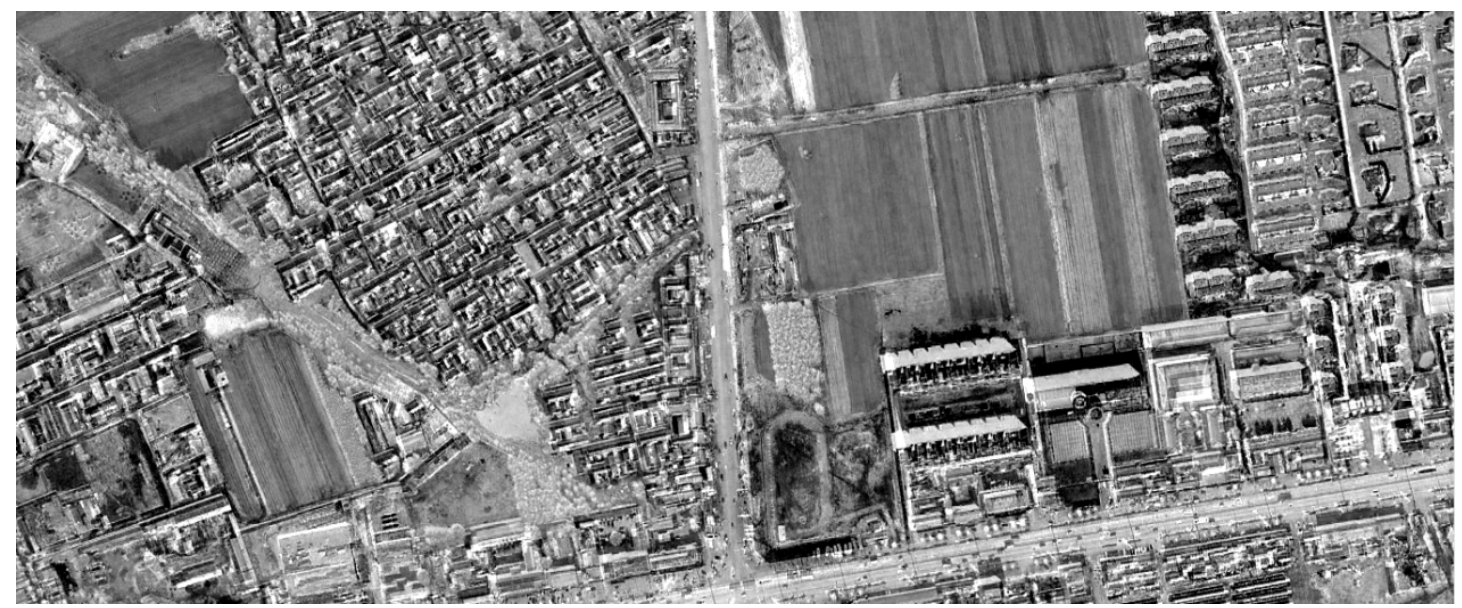

Figure 9: Figure 3 and Figure 4's different change file

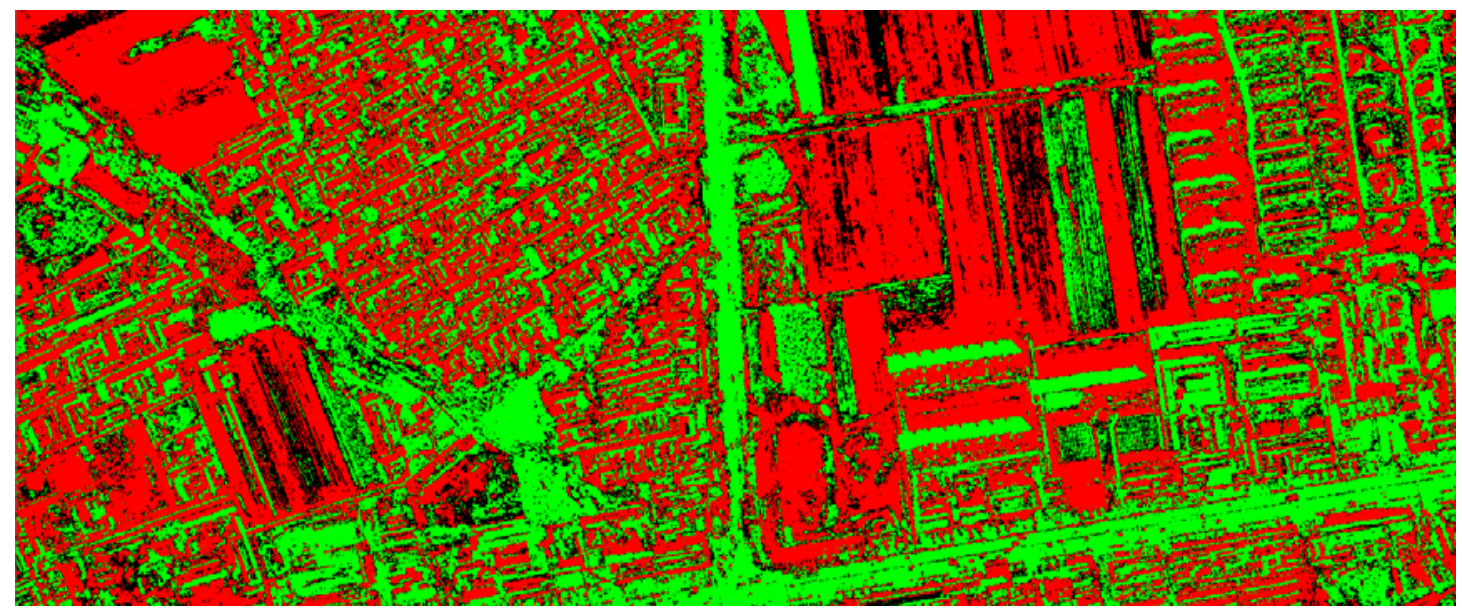

Figure 10: Figure 3 and Figure 4's different change file

Figure 9, Figure 10 are different change and highlight change files between year 2009 and year 2010 , respectively.

In Figure 9 the gray value of farmland area had a larger change, and in Figure 10 the gray value of farmland area increased. There was not much change in Figure 3 and Figure 4. The reasonable explanation for this phenomenon was: the farmland was not used in time, and then abandoned naturally.

Based on the change detection technology of multi-source remote sensing images, the 19 geographical coordinates in Xiadian fault zone of 2006-2011 were analyzed one by one. It could be deduced that in this period of time, the area of farmland in Xiadian was shrinking rapidly with more and more buildings and factories, green was better in some area, but there were also problems of farmland abandoned and time limit for a project delaying.

\section{Conclusions}

Based on the above analysis, the change detection method can be applied to monitor the land use situation of fault zone, which can show the change process of land use more directly and clearly. It provides data support for the next algorithm designing. If the technology can get more funds to support practical application, it will be conducive for local governments and relevant departments to 
understand the environmental changes, and can provide decision-making basis for better protection and improvement of the environment.

\section{Acknowledgements}

Two of Authors (Xiang Zhang, Maofa Wang) thank the financial support from Special Fund of Fundamental Scientific Research Business Expense for Higher School of Central Government: ZY20160106 )

\section{References}

[1] HE Fu-bing, BAI Ling-yan, WANG Ji-ming, et al. (2013) Deep Structure and Quaternary Activities of the Xiadian Fault Zone. Seismology and Geology, 35(3), 490-505.

[2] Maha Letchumy Balakeristanan, Md Azlin Md Said. (2012) Land Use Land Cover Change Detection Using Remote Sensing Application for Land Sustainability. International Conference on Fundamental and Applied Sciences, 425-430.

[3] Chen, J. Y., Q. J. Tian. (2007) Vegetation Classification Based on High-Resolution Satellite Image. Journal of Remote Sensing, 2, 221-227.

[4] J. Ma, X. J. Shan. (2000) An Attempt to Study Fault Activity Using Remote Sensing Technology-A Case of the Mani Earthquake. Seismology and Geology, 22(3), 210-215.

[5] Z. X. Li. (1993) The Study on Interpretation of Overthrust and Coal Searching Using Tm Image in the West of Liaoning Province. Journal of Remote Sensing, 8(1), 55-63.

[6] W. N. Yang. (2001) Quantitative Analysis of Remote Sensing Images on Tectonic Deformation and Stress Fields, Southern Sichuan, China. Journal of Remote Sensing, 5(1), 62-68.

[7] VIJAYARAJ V, CHARLES G O' HARA, GUNNAR A O, et al. (2005) Object and Feature-Space Fusion and Information Mining for Change Detection. IEEE International, 5(5), 131-135.

[8] NIEMEYER I, NUSSBAUM S. (2007) Change Detection Using the Object Features. IEEE International, 7, 2374-2377.

[9] HAZEL G. (2001) Object Level Change Detection in Spectral Imagery. IEEE Transactions on Geoscience and Remote Sensing, 39(3), 553-561. 